\title{
Texture Analysis of Polycrystalline Vaterite Spherulites
}

Fish otoliths, or ear bones, are comprised of the $\mathrm{CaCO}_{3}$ polymorphs (aragonite, calcite and vaterite), which can occur either alone or in combination. The polymorph phase abundance in an otolith depends on, as yet, unexplained genetic and environmental factors. Most fish otoliths are comprised of the densest $\mathrm{CaCO}_{3}$ polymorph, aragonite. Sturgeon otoliths, on the other hand, contain significant amounts of the rare and structurally enigmatic polymorph, vaterite. Sturgeon otoliths are frequently comprised of agglomerations of small microcrystalline vaterite spherulites ( $<100$ microns in diameter), that range in shape from nearly perfect spheres to oblate spheroids. These spherulites are similar to synthetic vaterite microspheres employed in microrheological systems used to measure properties of complex fluids in small fluid volumes. In these instruments, the birefringent vaterite microspheres have a rotation directed by a polarized laser light, and the diffusional rotation is relatable to the fluid viscosity. Whether synthesized or natural, the texture manifested in these microspheres will give clues to how vaterite nucleates and grows. Data were collected using a Rigaku XtaLAB PRO diffractometer equipped with graphite monochromated Mo Ka radiation, a Dectris Pilatus 200K detector, and the Rigaku Oxford Diffraction CrysAlisPro software. Texture analysis was done using MAUD 2.78 (Lutterotti 2017). Research conducted at ORNL's Spallation Neutron Source was sponsored by the Scientific User Facilities Division, Office of Basic Energy Sciences, U.S. Department of Energy.

Bryan Chakoumakos Oak Ridge National Laboratory chakoumakobc@ornl.gov
Brenda Pracheil
R. Seth Wood
Alison Loeppky
Gary Anderson
Ryan Koenigs
Ronald Bruch

